\title{
Economic Impact of Sectoral Transactions in Nigeria
}

\author{
Mfonobong O. Effiong1, Chukwuemeka U. Okoye ${ }^{2}$, Noble Jackson Nweze ${ }^{2}$ \\ ${ }^{1}$ Department of Agricultural Economics, University of Calabar, Calabar, Nigeria \\ ${ }^{2}$ Department of Agricultural Economics, University of Nigeria, Nsukka, Nigeria \\ Email:mfonnkang@yahoo.com
}

How to cite this paper: Effiong, M.O., Okoye, C.U. and Nweze, N.J. (2019) Economic Impact of Sectoral Transactions in Nigeria. Modern Economy, 10, 2283-2299. https://doi.org/10.4236/me.2019.1011143

Received: October 12, 2019

Accepted: November 25, 2019

Published: November 28, 2019

Copyright $\odot 2019$ by author(s) and Scientific Research Publishing Inc. This work is licensed under the Creative Commons Attribution International License (CC BY 4.0).

http://creativecommons.org/licenses/by/4.0/ (c) () Open Access

\begin{abstract}
Most applications of input-output (I-O) analysis to date have been to highlight inter-industry flows and to estimate the main aggregate national accounts, such as GDP, gross output and final demand categories. However, multiplier coefficients relating to output and income multipliers have hardly been explored especially in the Nigerian context. Sectors like agriculture, fishing, food \& beverages as well as mining/quarrying have particularly significant roles and their economic impacts can be quantified using Nigeria's I-O table. The study adopted a longitudinal design and utilized the $2015 \mathrm{I}-\mathrm{O}$ table comprising of twenty-six (26) sectors obtained from Eurostat database. This table was used to compile an inter-industry transaction table and Leontief matrix, which was then used to derive industry-wise Type I and Type II multipliers for the aforementioned sectors. Type I multiplier takes into account the direct and indirect effects while the Type II multiplier captured the induced effects in addition to the direct and indirect effects. Mining/quarrying as a single sector had a Type I multiplier of 1.80 and 2.17 for both output and income respectively and a Type II multiplier of 2.41 and 3.12 for both output and income respectively. Similarly, the fishing sectors were identified to have the highest contributions (2.11 and 2.89 as well as 2.22 and 3.19) in both Types I and II multipliers for both output and income respectively when compared with other sectors.
\end{abstract}

\section{Keywords}

Input-Output Analysis, Sectoral Transactions, Eurostat Database, Type I and II Multipliers, Nigeria

\section{Introduction}

Nigeria is one of Africa's largest economy known for its increased fishing, min- 
ing and agricultural activities. Owing to the increased population in the country, demand for products from these sectors has been increased over the years and has contributed immensely to nation's Gross Domestic Product [1].

The agricultural sector has been known to contribute about 20.85 percent to the nation's GDP (National Bureau of Statistics, 2018) [2]. The amount of value added measured in nominal terms was 5,288,339.21 million naira in the third quarter of 2018. Average annual growth rate of the mining industry had a GDP of about 8.71 percent increase at the end of 2018. The fishing sectors has also been known as a major contributor to Nigeria's GDP in last five (5) years following the protectionist trade measure in fish production (import quota) introduced since the first quarter of 2014 has stimulated the country's self-sufficiency through a 25 percent annual fish import cut [3].

Input-output analysis has been proven to be one of the most useful techniques to measure economic impacts, relating to both indirect and induced impacts, like the effect of given levels of final demand, for example, personal consumption expenditure, government expenditure, capital formation and exports. It is also possible to forecast the elements of the economy under different assumptions regarding the level of one or more of these indicators. This quantitative analysis first introduced by [4] is a top-down approach used to describe the general flow of goods and services in an economy, classified into various sectors. The total output multiplier for a sector measures the sum of the direct and indirect input requirements from all sectors needed to fulfill the final demand requirements of that sector. Multiplier effects which are associated with Keynes are defined as the change in equilibrium GDP divided by the change in investment [5]. The multiplier effect has been used as an argument for the efficacy of government expenditures to stimulate aggregate demand. One of the best-known results of input-output analysis is its ability to derive multipliers using supply and use sides of the national accounts [6].

This paper is aimed at estimating the economic impacts resulting from sectors like, mining/quarrying, fishing, food \& beverages and agriculture on the Nigerian economy using the input-output multiplier analysis. This also helps to predict the consequences of any planned and potential changes in the demand for the country's output. The study derived a Type I and Type II multipliers for the aforementioned sectors. The Type I multiplier measures the change in output in both sectors due to the change in final demand. It is the ratio between the change in gross output and the change in final demand. For example, as presented in Table 1, if $\$ 1$ additional demand for agricultural inputs generates \$ 2 additional gross industrial output then the Type I multiplier relating to the agricultural sector is equal to 2 . In other words, if the Type I agricultural multiplier is 2 , then for each $\$ 1$ additional demand for agricultural inputs would generate $\$ 2$ worth of additional gross output within the economy.

Specifically, this paper sets to describe the use of Type I and Type II multipliers to measure direct, indirect and induced effects from the fishing, min- 
ing/quarrying, food/beverage and agricultural sectors in Nigeria. The paper also highlights the important steps involved in deriving Type I and Type II multipliers from the I-O table to include the calculation of inter-industry transaction table and the Leontief matrix. The most recent I-O table was obtained for 2015 at aggregated level with 26 industries [7]. The reference year of 2015 means the multiplier coefficients need to be updated when more recent I-O tables are available.

\section{Review of Related/Empirical Literature}

Input-output analysis has been known as a veritable tool that addresses the following: multiplier effects of an investment program; environmental restrictions impact on prices; national accounting as well as its efficiency and dynamic performance [8]. However, application of input-output analysis to measure economic impacts for fishing, mining/quarrying, food/beverage and agricultural sectors has been limited. According to [9], output, employment and income multipliers have been known to be used to describe different economic impacts thus:

1) Output Multipliers

Output multiplier for an industry is defined as the ratio of output changes to a unit increase in final demand. This is, Changes in output

Final demand

\section{2) Employment Multipliers}

The employment multiplier expresses an estimate of the total employment attributable to the stimulus per man-year of employment.

\section{3) Income Multipliers}

This measures the change in income (wages, salaries, and profits, etc.) which occurs throughout the economy as a result of a change in final demand.

Related studies have been carried out by researchers using input-output analysis and multipliers to investigate economic impacts but dearth is evident in $\mathrm{Ni}$ geria. [10] used input-output tables to analyze the use of energy for transport purposes in Germany. He calculated energy necessities of transport-related final demand by means that of the Leontief-inverse connected to the energy information. He found that the energy necessities of transport-related final demand have truly big quicker than the energy consumption by transport as associate business.

On the other hand, [11] in his study suggested input-output multiplier analysis as one of the recommended techniques for assessing economic impacts of transportation projects. Their output multiplier coefficients (Type I) for transport services were 2.4 and 1.8 for larger and smaller state respectively [12].

\section{Methodology}

\subsection{Data Sources}

The latest available Nigeria's I-O table was the symmetric for year 2015 and was obtained from Eurostat database who considered all the "classical" drawbacks of 
the I-O approach (static, linear production function, no substitution or scale economy effects, infinite elasticity of supply) in its estimation and interpretation. It consisted of forty-six (46) sectors aggregated into twenty-six (26) sectors of economic activity, compiled following the industry-technology assumption, product-by-product, with total flows and valued at basic values at current prices.

\subsection{Model Specification}

1) The Theoretical Model

The income expenditure equality is given by:

$$
E=C+I+G+X-M
$$

where,

$E=$ expenditure measure of Gross Domestic Product $(G D P)$;

$C=$ consumption; $I=$ Investment;

$G=$ Government expenditure;

$X=$ Exports;

$M=$ Imports.

$C+I+G+X-M=$ components of final demand;

$C=$ Household consumption expenditure $(H C E)$;

$I=$ Fixed Capital Formation;

$G, X, \& M$ are as already defined.

Re-writing Equation (1), we have:

$$
E=G D P=C+I+G+X-M
$$

In terms of production, $G D P$ value is given as:

$$
G O-I C=G D P=C+I+G+X-M
$$

where,

$G O=$ Gross Output;

$I C=$ intermediate consumption

Multiplying GO-IC by Gross output and simplifying we have:

$$
G O\left(1-\frac{G O}{I C}\right)=G D P
$$

But, $a=\frac{G O}{I C}$, by substitution we have:

$$
G O(1-a)=G D P
$$

In terms of GO, we have,

$$
G O=(1-a)^{-1} V A
$$

where,

$$
V A=G D P
$$

$(1-a)^{-1}=$ Leontief Inverse proportion of

$\frac{G O}{I C}=$ Proportion of intermediate consumption in the gross output which is 
also referred to as the technical coefficient matrix in the Input-output analysis.

By inversion, the symmetric matrix, $(1-a)$ is transformed to get the asymmetric input-output table using the supply and use table.

Equation (6) forms the basis for the multiplier analysis. The column sum of the Leontief Inverse which is also known as the total requirement matrix, shows the input requirements for a unit increase in the final demand for a given industry, called the multiplier coefficient. These input requirements commonly referred to as "backward linkages" measure the impact on the supplier industries of a unit increase in final demand [13].

However, the following steps are involved in the transformation of the asymmetric matrices (supply and use tables) to an input-output table viz:

\section{2) The Empirical Model}

\section{Use and supply tables}

Suppose an economy with " $t$ " number of products and "I" number of industries. The relationship between the use of products by industries and end users are presented in Table 1.

where,

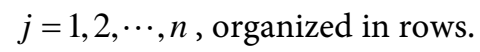

Industries are denoted by $\operatorname{Ind}(\mathrm{k})$ where,

$k=1,2, \cdots, n$, organized in columns.

The columns represent the value of the intermediate consumption for the corresponding industry, which uses various products by a particular industry. Similarly, the rows represent the value-added components of each industry. The gross output of each industry is given by the sum of the total intermediate consumption.

On the other hand, Table 2 presents the supply of products to various industries.

Table 1. (a) Use of products by industries and end users (use table); (b) data used for analysis.

(a)

\begin{tabular}{|c|c|c|c|c|c|c|c|c|c|c|c|}
\hline & & \multicolumn{4}{|c|}{ Industry use } & \multicolumn{5}{|c|}{ End users } & \multirow{2}{*}{$\begin{array}{c}\text { Products gross } \\
\text { output }\end{array}$} \\
\hline & & Ind (1) & Ind (2) & .. & Ind (n) & $\mathrm{HC}$ & GP & INV & Exp & $\operatorname{Imp}$ & \\
\hline & Com (1) & $\mathrm{i} 1,1$ & $\mathrm{i} 1,2$ & .. & $\mathrm{i} 1, \mathrm{n}$ & $\mathrm{hc}_{1}$ & $\mathrm{gp}_{1}$ & inv $_{1}$ & $\exp _{1}$ & $\mathrm{imp}_{1}$ & go $(\mathrm{com})_{1}$ \\
\hline \multirow{3}{*}{ Product } & Com (2) & $\mathrm{i} 2,1$ & $\mathrm{i} 2,2$ & .. & $\mathrm{i} 1, \mathrm{n}$ & $\mathrm{hc}_{2}$ & $\mathrm{gp}_{2}$ & $\operatorname{inv}_{2}$ & $\exp _{2}$ & $\mathrm{imp}_{2}$ & go $(\mathrm{com})_{2}$ \\
\hline & .. & .. & .. & .. & .. & .. & .. & .. & .. & .. & .. \\
\hline & $\operatorname{Com}(\mathrm{m})$ & $\mathrm{im}, 1$ & im, 2 & .. & im,n & $\mathrm{hc}_{\mathrm{m}}$ & $\mathrm{gp}_{\mathrm{m}}$ & $\operatorname{inv}_{\mathrm{m}}$ & $\exp _{\mathrm{m}}$ & $\mathrm{imp}_{\mathrm{m}}$ & go $(\mathrm{com})_{\mathrm{m}}$ \\
\hline \multirow{3}{*}{ GDP } & Compensation of employees & $\mathrm{iw}_{1}$ & $\mathrm{w}_{2}$ & .. & $\mathrm{wn}$ & & & & & & \\
\hline & Operating surplus & $\mathrm{ops}_{1}$ & $\mathrm{ops}_{2}$ & .. & $\mathrm{ops}_{\mathrm{n}}$ & & & & & & \\
\hline & Taxes on products & $\operatorname{taxp}_{1}$ & $\operatorname{taxp}_{2}$ & .. & $\operatorname{taxp}_{\mathrm{n}}$ & & & & & & \\
\hline & Industry Gross Output & go (ind) ${ }_{1}$ & go (ind) $)_{2}$ & .. & go (ind) $)_{n}$ & & & & & & \\
\hline
\end{tabular}

Source: Authors conceptualization. NB: Products are denoted by Com (j). 
(b)

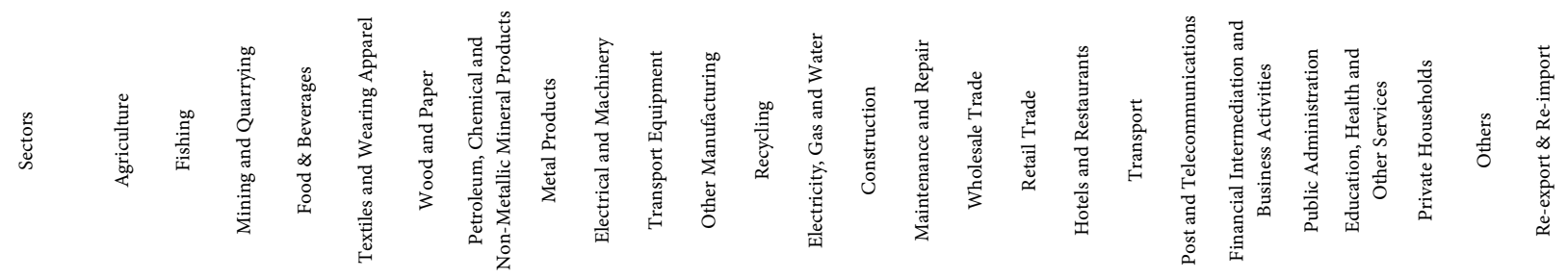

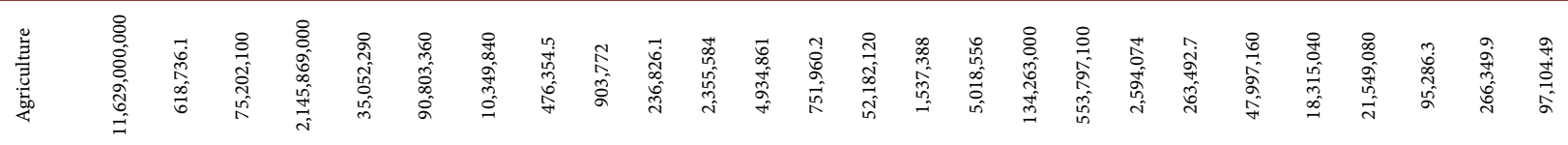

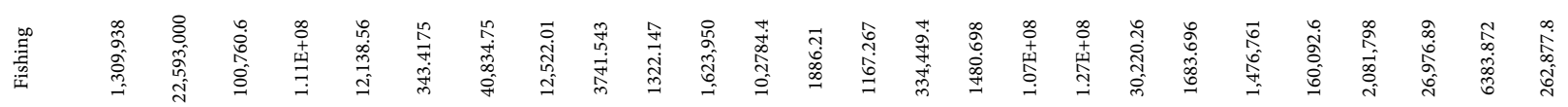

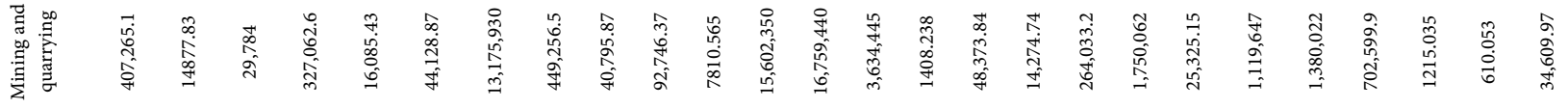

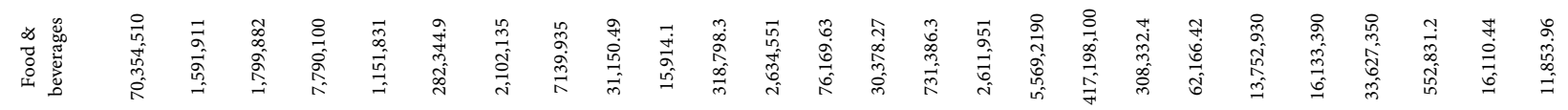

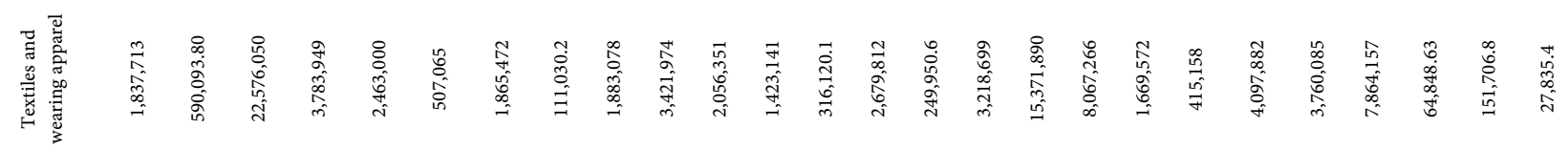

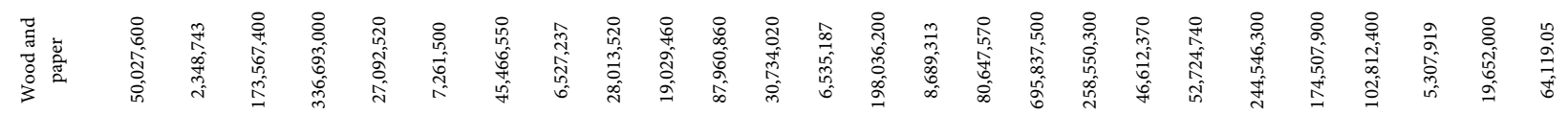

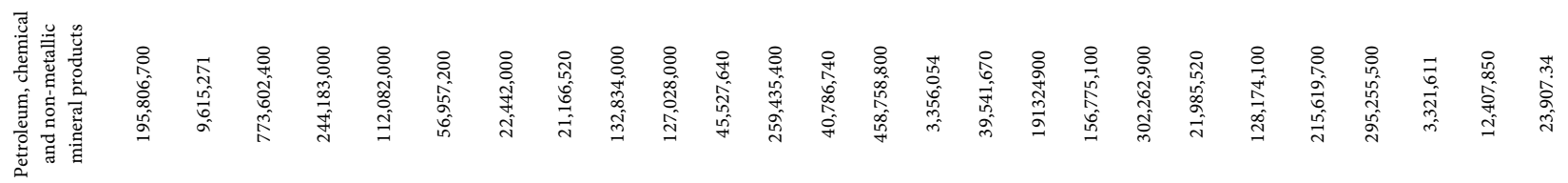

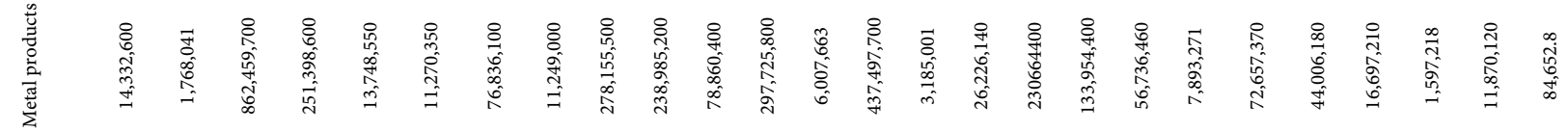

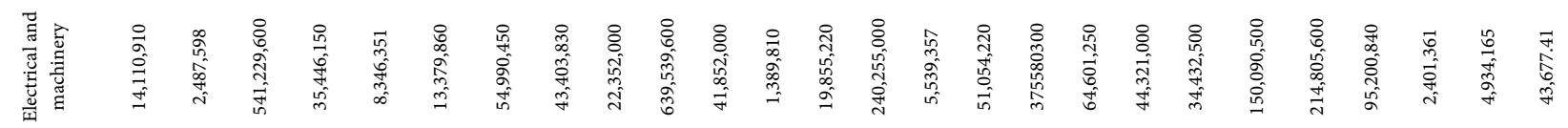

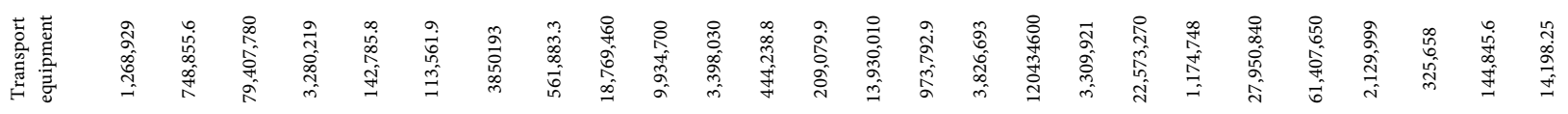

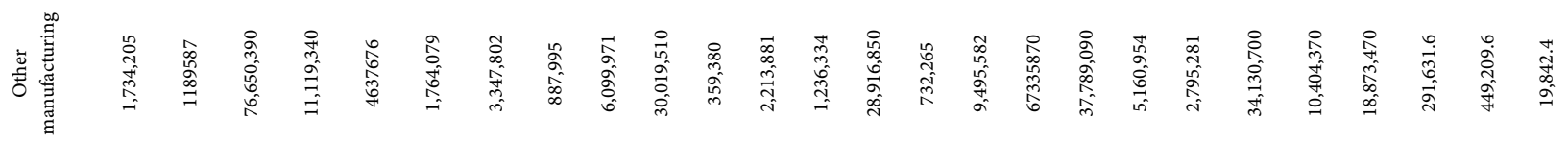

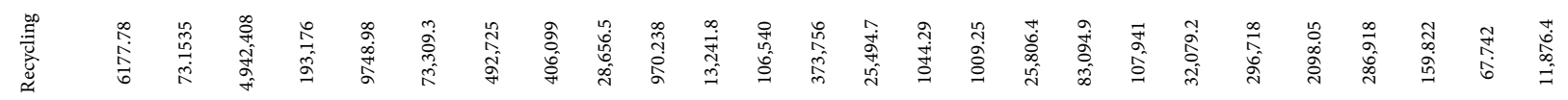

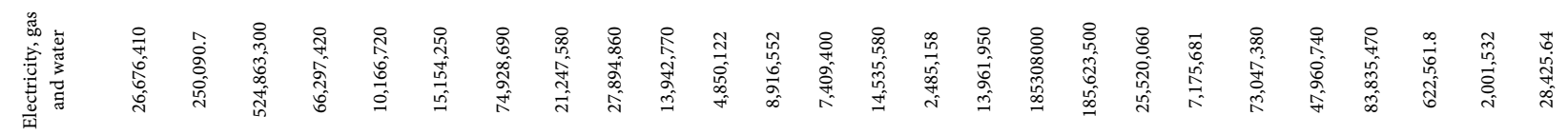




\section{Continued}

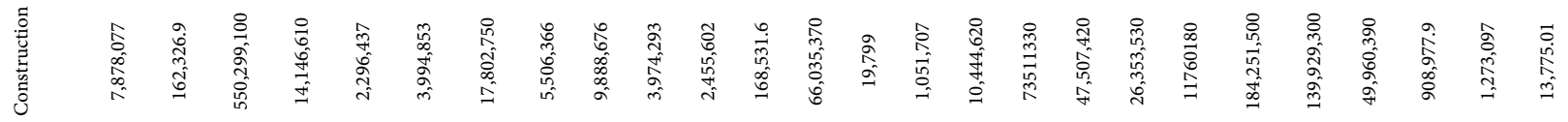

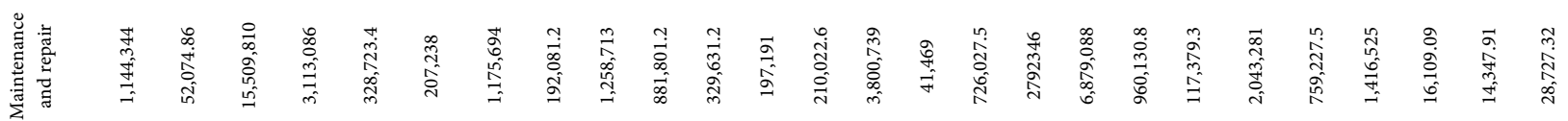

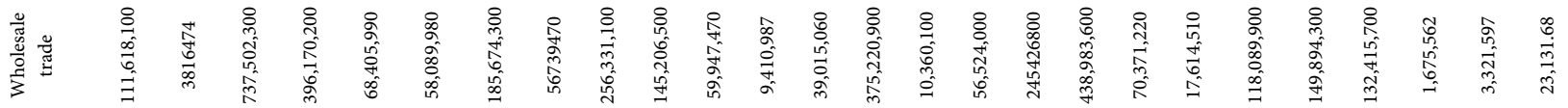

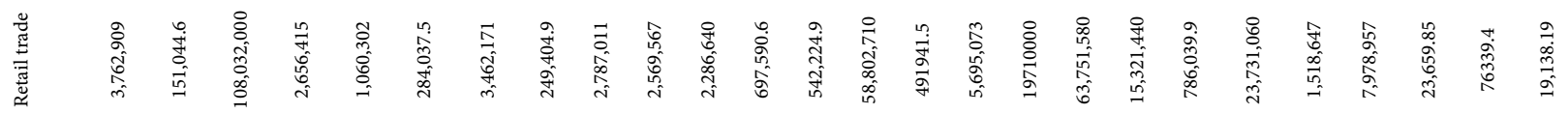

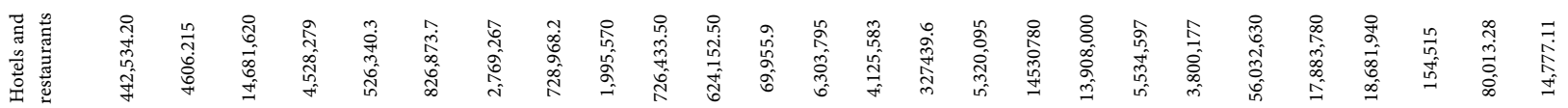

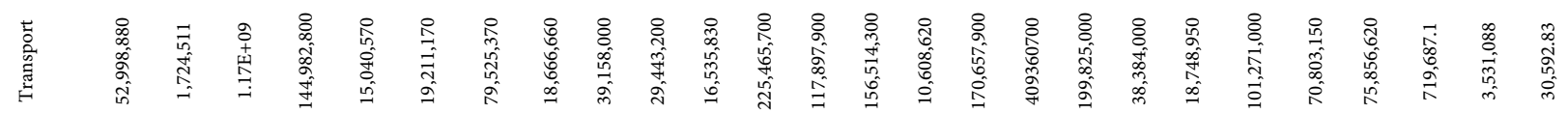

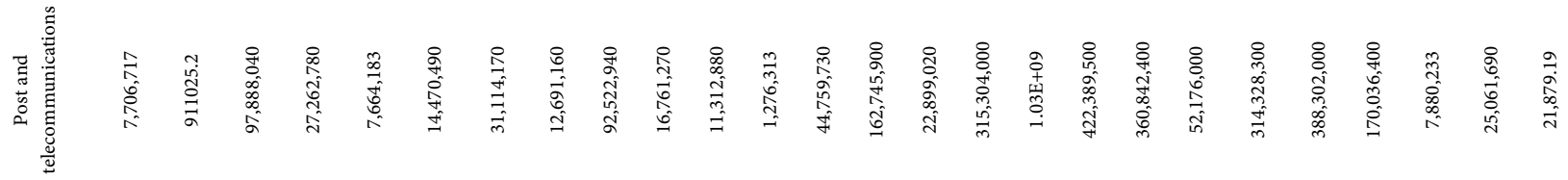

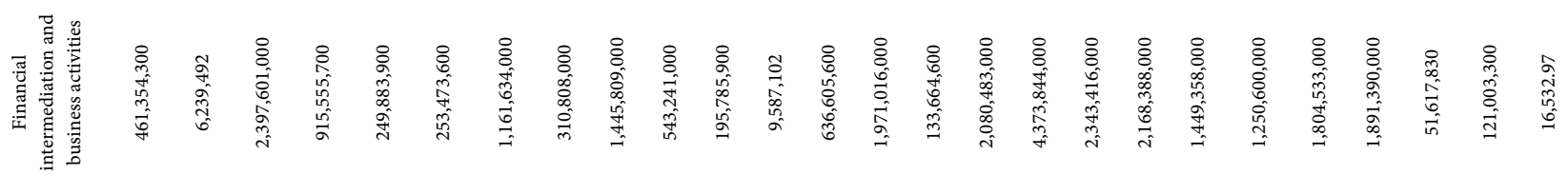

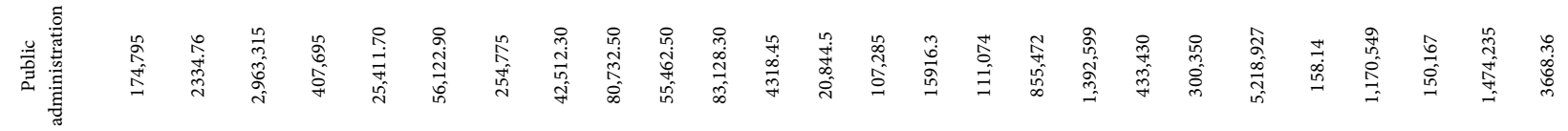

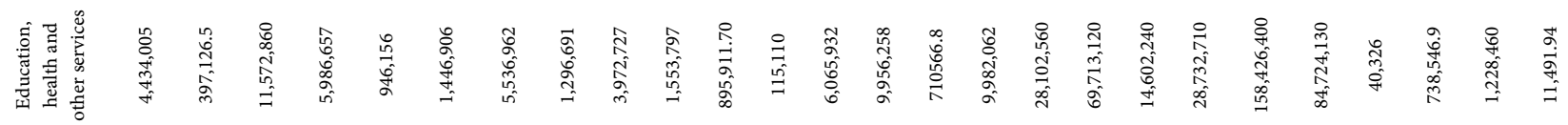

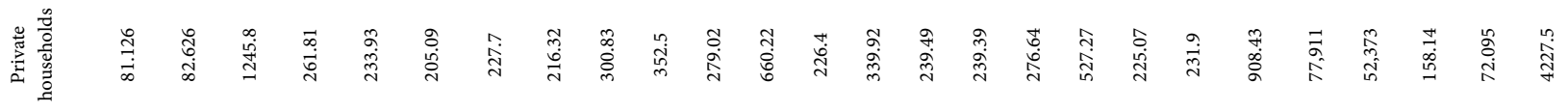

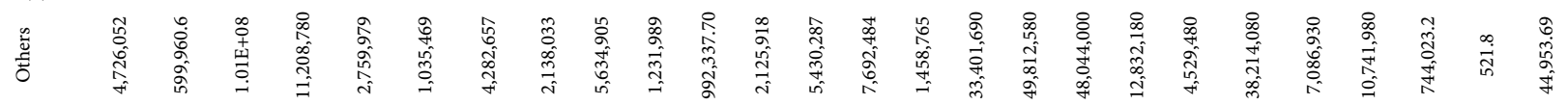

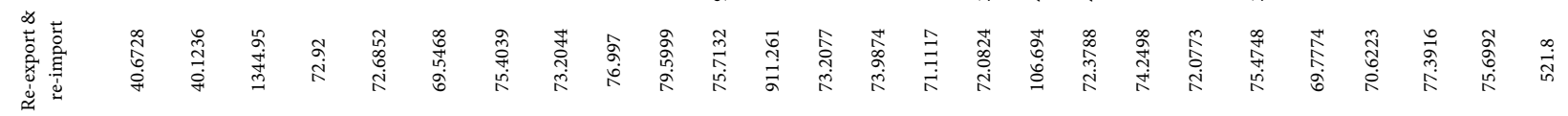

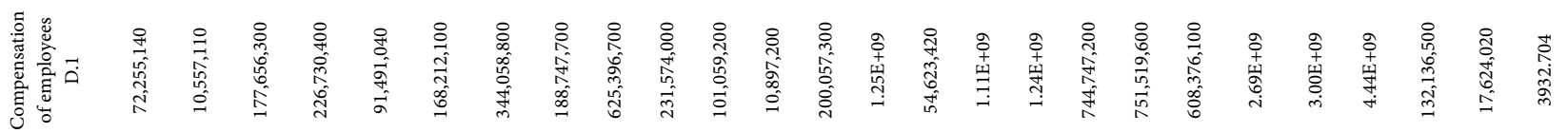

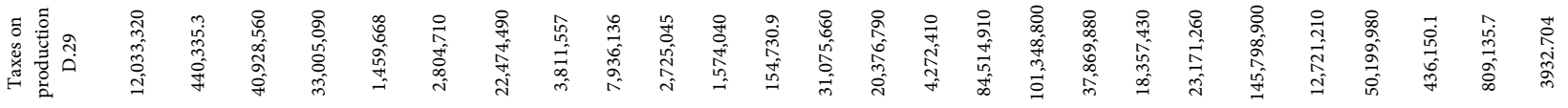

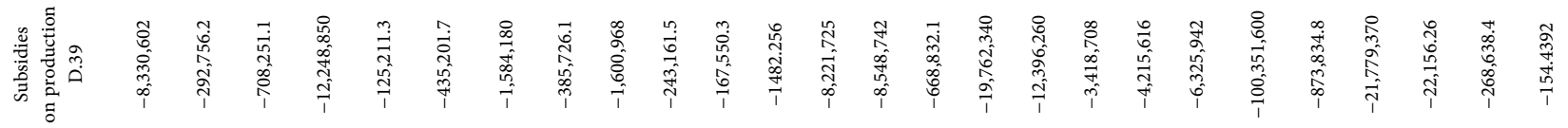


Continued

\begin{tabular}{|c|c|c|c|c|c|c|c|c|c|c|c|c|c|c|c|c|c|c|c|c|c|c|c|c|c|c|}
\hline 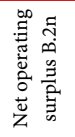 & 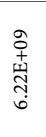 & 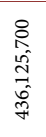 & $\begin{array}{l}\stackrel{\circ}{7} \\
\text { 岃 } \\
\stackrel{-}{-}\end{array}$ & $\begin{array}{l}\text { oे } \\
+ \\
\text { 㟧 } \\
\text { co }\end{array}$ & $\begin{array}{l}\text { 古 } \\
\text { 离 } \\
\text { 促 }\end{array}$ & 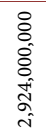 & $\begin{array}{l}\stackrel{o}{+} \\
\text { 离 } \\
\stackrel{\sigma}{\sigma}\end{array}$ & $\begin{array}{l}\text { oे } \\
\text { 古 } \\
\text { } \\
\text { iv }\end{array}$ & 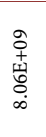 & $\begin{array}{l}\text { oे } \\
\text { + } \\
\text { 岕 } \\
\text { r. } \\
\text { r. }\end{array}$ & 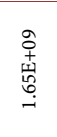 & 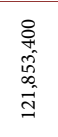 & 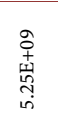 & $\begin{array}{l}\text { oे } \\
\text { 㟔 } \\
\text { m. } \\
\text { o. }\end{array}$ & 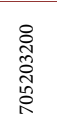 & $\begin{array}{l}\stackrel{0}{+} \\
\text { 嵒 } \\
\stackrel{9}{-}\end{array}$ & 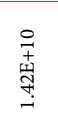 & 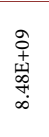 & 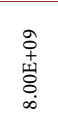 & 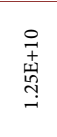 & $\begin{array}{l}\overrightarrow{7} \\
\text { ज्ञ } \\
\text { ㄱ }\end{array}$ & $\begin{array}{l}\stackrel{9}{+} \\
\text { 离 } \\
\text { m) }\end{array}$ & 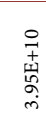 & & & 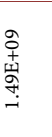 \\
\hline 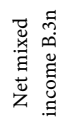 & $\begin{array}{l}0 \\
0 \\
\hat{\Xi} \\
0 \\
\hat{0} \\
\hat{0}\end{array}$ & 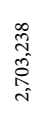 & 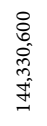 & 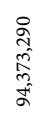 & 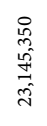 & 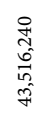 & 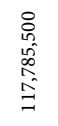 & 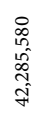 & 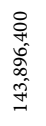 & $\begin{array}{l}0 \\
0 \\
0 \\
\hat{i} \\
\hat{n} \\
0 \\
0 \\
0\end{array}$ & 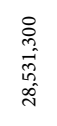 & 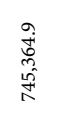 & 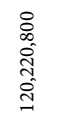 & 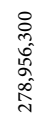 & $\begin{array}{l}0 \\
\hat{n} \\
0 \\
0 \\
0 \\
\hat{\imath}\end{array}$ & 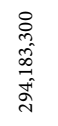 & $\begin{array}{l}8 \\
\infty \\
0 \\
\hat{N} \\
\hat{N} \\
\tilde{~}\end{array}$ & 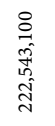 & 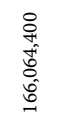 & 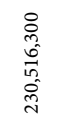 & 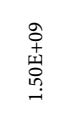 & 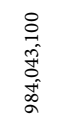 & $\begin{array}{l}\text { oे } \\
\text { 复 } \\
\text { ت }\end{array}$ & & & \\
\hline 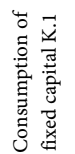 & $\begin{array}{l}0 \\
\text { nn } \\
w \\
0 \\
0 \\
0 \\
n\end{array}$ & 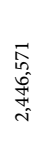 & $\begin{array}{l}8 \\
0 \\
0 \\
0 \\
0 \\
\hat{0} \\
\hat{~}\end{array}$ & 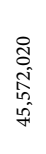 & $\begin{array}{l}\text { ले } \\
0 \\
\infty \\
0 \\
\stackrel{2}{\cong}\end{array}$ & $\begin{array}{l}\stackrel{2}{1} \\
0 \\
0 \\
\hat{+}\end{array}$ & 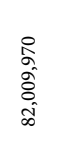 & 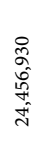 & $\begin{array}{l}\text { a } \\
\text { aे } \\
\text { da }\end{array}$ & 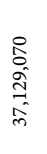 & 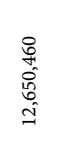 & $\begin{array}{l}0 \\
\infty \\
\infty \\
\infty \\
\stackrel{0}{-1}\end{array}$ & 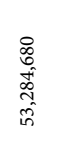 & $\begin{array}{l}8 \\
\infty \\
0 \\
0 \\
\circ \\
0 \\
0 \\
0\end{array}$ & 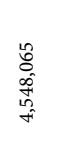 & 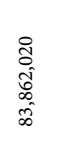 & $\begin{array}{l}8 \\
\stackrel{8}{0} \\
\overrightarrow{3} \\
\overrightarrow{0} \\
\stackrel{0}{=}\end{array}$ & \begin{tabular}{l} 
స్ \\
ô. \\
\multirow{0}{*}{} \\
$\hat{i}$
\end{tabular} & 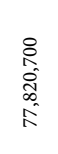 & 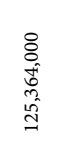 & 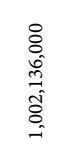 & 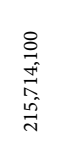 & 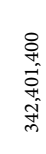 & & & \\
\hline
\end{tabular}

Source: Eurostat Database.

Table 2. Supply of products to industries (supply table).

\begin{tabular}{|c|c|c|c|c|c|c|}
\hline & & \multicolumn{4}{|c|}{ Sectors } & \multirow{2}{*}{$\begin{array}{c}\text { Products gross } \\
\text { output }\end{array}$} \\
\hline & & $\operatorname{Sec}(1)$ & $\operatorname{Sec}(2)$ & .. & $\operatorname{Sec}(m)$ & \\
\hline \multirow{4}{*}{ Product } & Com (1) & $\mathrm{s} 1,1$ & & .. & $\mathrm{s} 1, \mathrm{n}$ & gross $(\mathrm{com})_{1}$ \\
\hline & Com (2) & $\mathrm{s} 2,1$ & & .. & $\mathrm{s} 2, \mathrm{n}$ & gross $(\mathrm{com}) 2$ \\
\hline &.. &.$\cdot$ &.$\cdot$ &.. &.. &.. \\
\hline & $\operatorname{Com}(\mathrm{m})$ & $\mathrm{sm}, 1$ & & .. & $\mathrm{sm}, \mathrm{n}$ & $\operatorname{gross}(\mathrm{com}) \mathrm{m}$ \\
\hline \multicolumn{2}{|c|}{ Sectors Gross Output } & $\begin{array}{l}\text { Gross } \\
(\mathrm{sec})_{1}\end{array}$ & $\begin{array}{l}\text { Gross } \\
(\mathrm{sec})_{2}\end{array}$ &.. & $\operatorname{Gross}(\mathrm{sec})_{\mathrm{n}}$ & \\
\hline
\end{tabular}

Authors' conceptualization.

Each row shows the value of products supplied to each industry while the columns represent the industry gross output for each sector. The total gross output of products in the use table should be equal to those in the supply table. Also, the industry gross outputs in the use tables should be equal to those in the supply table. This equality characteristic forms the basis in national income/expenditure accounting.

\section{3) The Input-Output Table}

As presented in Table 1 and Table 2, the use and supply tables are used to calculate the use and supply proportions, technical coefficients and the inter-industry or inter-product transaction tables. The inter-industry or inter-product transaction tables are important for compiling the input-output tables. A typical input-output table is presented in Table 3. An input-output table consists of an inter-product transaction table (the shaded area), the final demand matrix and the value added or GDP components (measured using production method).

The shaded area represents the inter-industry coefficients where output of an industry can be used as input in other industries while input of an industry can be used to produce a good. For example, industry $A_{1,2}$ implies that, industry 1 supplies input to industry 2 for use its production process while industry 2 is the purchaser or user of the inputs. This table is the matrix required to calculate the Leontief matrix and the Type I \& II multipliers are presented as follows: 
Table 3. Input-output table.

\begin{tabular}{|c|c|c|c|c|c|c|c|c|c|c|c|}
\hline & & \multicolumn{4}{|c|}{ Industry/sectors } & \multicolumn{5}{|c|}{ Final users } & \multirow{2}{*}{$\begin{array}{c}\text { Sectors } \\
\text { gross output }\end{array}$} \\
\hline & & Sector (1) & Sector (2) & $\ldots$ & Sector (n) & $\mathrm{HC}$ & Govt & Invt. & Exp. & Imp. & \\
\hline \multirow[t]{4}{*}{ Industry } & $\operatorname{Sec}(1)$ & $A_{1,1}$ & $\mathrm{~A}_{1,2}$ & $\ldots$ & $\mathrm{A}_{1, \mathrm{n}}$ & $\mathrm{Hc}_{1}$ & Govt $_{1}$ & Invt $_{1}$ & $\operatorname{Exp}_{1}$ & $\mathrm{imp}_{1}$ & Gross $(\mathrm{sec})_{1}$ \\
\hline & $\operatorname{Sec}(2)$ & $\mathrm{A}_{2,1}$ & $\mathrm{~A}_{2,2}$ & $\cdots$ & $\mathrm{A}_{2, \mathrm{n}}$ & & & & & $\mathrm{imp}_{2}$ & Gross $(\mathrm{sec})_{2}$ \\
\hline & & $\ldots$ & $\ldots$ & $\ldots$ & $\ldots$ & & & & & $\ldots$ & $\ldots$ \\
\hline & $\operatorname{Sec}(n)$ & $A_{n, 1}$ & $\mathrm{~A}_{\mathrm{n}, 2}$ & $\ldots$ & $A_{n, n}$ & & & & & $\mathrm{imp}_{\mathrm{n}}$ & Gross $(\mathrm{sec})_{3}$ \\
\hline \multirow[t]{3}{*}{ Value added } & $\begin{array}{l}\text { Compensation of } \\
\text { employee (wages) }\end{array}$ & W1 & W2 & $\ldots$ & $\mathrm{W}_{3}$ & & & & & & \\
\hline & Operating surplus & $\mathrm{Ops}_{1}$ & $\mathrm{Ops}_{2}$ & $\ldots$ & $\mathrm{Ops}_{3}$ & & & & & & \\
\hline & Taxes on products & $\operatorname{Taxp}_{1}$ & $\operatorname{Taxp}_{2}$ & $\ldots$ & $\mathrm{Taxp}_{3}$ & & & & & & \\
\hline Sectors Gross output & & Gross $(\mathrm{sec})_{1}$ & Gross $(\mathrm{sec})_{2}$ & $\ldots$ & Gross $(\mathrm{sec})_{3}$ & & & & & & \\
\hline
\end{tabular}

NB: HC = household consumption, Govt = government expenditure, Invt $=$ investment, Exp $=$ exports, Imp $=$ imports, Sec $=$ sectors, Taxp $=$ taxes on products, Ops = operating surplus, $\mathrm{W}=$ wages.

\section{Type I and II multipliers derivation}

In line with the UN guidelines [14] [7] there are five (5) steps involved in these derivations thus:

Step 1: Calculate from use and supply tables, the use and supply proportions;

Step 2: Calculate inter-product transaction table;

Step 3: Calculate Leontief matrix;

Step 4: Derivation of the multipliers;

Step 5: Validation of the empirical model.

\section{Step 1: The Use and Supply Proportions}

\section{Use proportions:}

Industry-by-industry use proportions are obtained by dividing each cell entry in the use table by industry gross output in the final row of the use table. We denote intermediate consumption and the value added parts of the use matrix as $i(j+v, k)$.

where,

$V=$ number of rows in value added part of the use table.

But, $G(1, k)=$ Industry gross output.

Then,

$$
B(j+v, k)=\frac{U(j+v, k)}{G(1, k)}
$$

Equation (7) represents the use proportions matrix comprising, intermediate consumption and value-added components.

The use proportion matrix with only intermediate consumption is given by:

$$
B(j, k)=\frac{U(j, k)}{G(1, k)}
$$

Each column in Equation (7) represents the proportion of use by each indus- 
try, having a column sum of use proportions to be equal to 1 .

\section{Supply proportions:}

Industry-by-industry supply proportions are obtained by dividing each cell entry by row sum as given below. Suppose the supply matrix is denoted by $M(j$, $k$ ). Gross output of products is a column vector and given by $Q(j, 1)$.

Then the supply proportions matrix is:

$$
D(j, k)=\frac{M(j, k)}{Q(j, 1)}
$$

Notice that row sum is equal to 1 , which means that each cell shows the proportion of supply of each product to a particular industry.

\section{Step 2: Inter-industry transaction table}

This is presented in two (2) different symmetric transaction tables viz:

a) Industry-by-industry transaction table;

b) Product-by-product transaction table.

The industry-by-industry transaction table is also known as inter-industry transaction table with an equal number of industries (in both rows and columns). The product-by-product transaction table is with an equal number of products (in both rows and columns). However, for this paper used the industry-by-industry transaction table to analyze the industry demand and the industry output, because of its proximity to the statistical sources and the actual market transactions [7].

The general transaction table is done using the use and supply proportions matrix.

Note: Intermediate consumption in use and supply proportions matrices have $m$ number of rows (products) and $n$ number of columns (industries). Hence, $m \neq$ $n$ represents rectangular matrices. Use and supply proportions matrices are as shown in Equations (8) and (9) and are used to calculate the technical coefficient matrix.

Using the Inter-industry transaction table, we obtained the industry-by-industry technical coefficients matrix as follows.

$$
a(m, m)=D^{\prime}(m, n) B(n, m)
$$

where,

$$
D^{\prime}(m, n)=\text { transpose of } D(n, m) .
$$

NB: Number of columns in the first matrix, $D^{\prime}(m, n)$ equals number of rows in the second matrix, $B(n, m)$. The resulting matrix denoted by $a(m, m)$ is called the industry-by-industry technical coefficient matrix. Each cell in this matrix represents the proportion of transaction from one industry to another industry, while the diagonal shows the transaction within one particular industry. We obtain the inter-industry transaction table by multiplying the technical coefficients matrix by a diagonal matrix representing industry gross output denoted by diag. $[Q(m, m)]$. The resulting inter-industry transaction table is denoted by $A(m, m)$. 


$$
A(m, m)=a(m, m) \operatorname{diag}[Q(m, m)]
$$

where,

$A(m, m)=$ symmetric matrix of size $m$ by $m$. Each cell in this matrix represents the value of transaction in dollars from one industry to another industry.

$\mathrm{D}^{\prime} A(k, k) \mathrm{s}=$ transaction within any particular industry.

\section{Step 3: Derivation of the Leontief inverse}

In Equation (6), the Leontief inverse matrix is presented as:

$$
L(m, m)=[I(m, m)-a(m, m)]^{-1}
$$

where,

$I(m, m)$ is an identity matrix of size $m$ by $m$.

Leontief inverse is obtained by:

Technical coefficients matrix $a(k, k)$ minus identity matrix $I(k, k)$.

By inversion, we have,

$L(k, k)$, which represents the Leontief matrix.

\section{Step 4: Derivation of Type I and II multipliers \\ For Type I multipliers}

Multiplier coefficients which represent the column sum of the Leontief inverse is given by:

$$
\alpha(i)=\sum_{i=1}^{n} L(i, k)
$$

where,

$$
\alpha(I)=\text { multiplier coefficient for any given industry. }
$$

\section{For Type II multipliers}

By introducing the household consumption ( $\mathrm{HC})$ sector as the $(k+1)^{\text {th }}$ column and employee income (compensation of employees) as $(k+1)^{\text {th }}$ row of the interindustry transaction table, the product-wise household consumption is transformed into the industry-wise household consumption by:

$$
H C(m, 1)=D^{\prime}(m, n) H C(n, 1)
$$

where,

$H C(n, 1)=$ column vector of HC (in terms of demand for products obtained from the use table);

$H C(m, 1)=\mathrm{HC}$ column vector (in terms of demand for industries).

$B u t, H C(m, 1)$ is added as the $(m+1)^{t h}$ column of the inter-industry transaction table which is the compensation of employees expressed in terms of industries as the row vector $\operatorname{COE}(1, m)$.

The new inter-industry transaction table now becomes $A(m+1, m+1)$.

As a follow-up, technical coefficients matrix from the new inter-industry transaction table is given thus:

$$
a(m+1, m+1)=\frac{A(m+1, m+1)}{Q(1, m+1)}
$$


where,

$A(m+1, m+1)=$ Individual columns;

$Q(1, m+1)=$ row vector of industry gross outputs;

$a(m+1, m+1)=$ technical coefficients matrix with an additional row for compensation of employees and an additional column for $H C$.

\section{Step 5: Validation of the empirical model}

This is done to ascertain the validity of the empirical exercise by re-estimating the gross output, intermediate consumption and value added using the model and then comparing them with the actual values. The estimated Leontief inverse is multiplied by the actual values for final demand to obtain the estimated values thus:

From Equation (6),

$$
G O=(L)(F D)
$$

where,

$G O=$ estimated gross output;

$L=$ estimated Leontief matrix;

$F D=$ actual total final demand.

The results of the model validation exercise are presented in Table 4.

\section{Results and Discussions}

The components of the final demand comprising, household consumption, non-profit institution serving households, government consumption, gross fixed capital formation and changes in inventories. are classified based on the individual industries. Table 5 summarizes the two (2) sets of industries: 1) Three industries representing only the agricultural sector (agriculture, fishing, food/ beverage); and 2) one industry representing only mining sector (mining/quarrying).

The components of the final demand give an indication of the significance of each component in the total final demand of each industry. For example, total final demand of agriculture in 2015 consists of 75 percent Household consumption, 25 percent non-profit institution serving households, 0.12 percent government consumption, 0.49 percent gross fixed capital formation and 0.09

Table 4. Results of the model validation exercise.

\begin{tabular}{cccc}
\hline & & Estimated total (\$ million) & Percentage (\%) \\
\hline $\begin{array}{c}\text { Industry by } \\
\text { industry method }\end{array}$ & Intermediate consumption & $106,619,721$ & 52.28 \\
& Value added & $96,744,498$ & 47.44 \\
\hline
\end{tabular}

Note: Actual totals are sourced from 2010 Nigeria's supply and use tables; This confirms a high level of accuracy of the empirical model. 
percent related to changes in inventories. Household consumption expenditure represents the largest proportion of the final demand. On the other hand, changes in inventories in terms of exports and imports are of particular importance to the agricultural sector.

Table 6 presents components of value added with the contributions of agricultural, fishing, food/beverage and mining industries to total GDP in 2015. It is also observed that approximately 1.13 percent of total compensation of employees is paid to employees in the agricultural sector which is relatively lower compared to the food/beverage industry (having 2.69 percent). The net operating surplus (profit) was highest in the agricultural industry (having about 97.01 percent) compared to other industries. The value-added components of the industries were found to be highest in the mining/quarrying industry having about $\$ 14,649,226,208.9$ million.

Table 5. Industry-wise final demand and gross output-2015. (Percentages are in parenthesis).

\begin{tabular}{|c|c|c|c|c|c|c|c|c|}
\hline & Industry & $\begin{array}{l}\text { Household } \\
\text { final } \\
\text { consumption }\end{array}$ & $\begin{array}{l}\text { Non-profit } \\
\text { institutions } \\
\text { serving } \\
\text { households }\end{array}$ & $\begin{array}{l}\text { Government } \\
\text { final } \\
\text { consumption }\end{array}$ & $\begin{array}{l}\text { Gross fixed } \\
\text { capital } \\
\text { formation }\end{array}$ & $\begin{array}{l}\text { Changes in } \\
\text { inventories }\end{array}$ & $\begin{array}{l}\text { Total final } \\
\text { demand }\end{array}$ & Gross output \\
\hline 1 & Agriculture & $\begin{array}{c}3,043,598,000 \\
(74.78)\end{array}$ & $\begin{array}{l}997,578,500 \\
(24.51)\end{array}$ & $\begin{array}{l}5,074,180 \\
(0.12)\end{array}$ & $\begin{array}{l}20,341,830 \\
\quad(0.49)\end{array}$ & $\begin{array}{c}3,640,730 \\
(0.09)\end{array}$ & $\begin{array}{c}4,070,233,240 \\
(100)\end{array}$ & $19,204,942,655.41$ \\
\hline 2 & Fishing & $\begin{array}{c}131,415,700 \\
(81.98)\end{array}$ & $\begin{array}{l}25,103,830 \\
(15.66)\end{array}$ & $\begin{array}{c}2,514,548 \\
(1.57)\end{array}$ & $\begin{array}{l}8644.47 \\
(0.005)\end{array}$ & $\begin{array}{c}1,243,550 \\
(0.78)\end{array}$ & $\begin{array}{c}160,286,272.47 \square \\
(100)\end{array}$ & $567,250,260.9$ \\
\hline 3 & $\begin{array}{l}\text { Food and } \\
\text { beverages }\end{array}$ & $\begin{array}{c}14,812,110,000 \\
(68.69)\end{array}$ & $\begin{array}{c}6,744,033,000 \\
(31.28)\end{array}$ & $\begin{array}{l}4644.285 \\
(0.000022)\end{array}$ & $\begin{array}{l}8007.873 \\
(0.000037)\end{array}$ & $\begin{array}{c}5,934,145 \\
(0.028)\end{array}$ & $\begin{array}{c}21,562,089,797.158 \\
(100)\end{array}$ & $322,729,713,512.514$ \\
\hline 4 & Mining/quarrying & $\begin{array}{l}137,691,400 \\
(85.93)\end{array}$ & $\begin{array}{l}15,892,030 \\
(9.92)\end{array}$ & $\begin{array}{c}1,543,437 \\
(0.96)\end{array}$ & $\begin{array}{c}2,411,855 \\
(1.51)\end{array}$ & $\begin{array}{c}2,706,671 \\
(1.69)\end{array}$ & $\begin{array}{l}160,245,393 \square \\
(100)\end{array}$ & $587,920,334.78$ \\
\hline & $\begin{array}{l}\text { Total of all } \\
\text { industries }\end{array}$ & $\begin{array}{c}15,111,652,900 \\
(50.90)\end{array}$ & $\begin{array}{c}7,782,607,360 \\
(26.22)\end{array}$ & $\begin{array}{c}6,753,165,165 \\
(22.75)\end{array}$ & $\begin{array}{c}22,770,337.343 \\
(0.077)\end{array}$ & $\begin{array}{c}13,525,096 \\
(0.046)\end{array}$ & $\begin{array}{c}29,683,720,858.343 \\
(100)\end{array}$ & $43,089,826,763.604$ \\
\hline
\end{tabular}

Computation from Eurostat database.

Table 6. Components of value added-2015 (\$ million) (Percentages are in parenthesis).

\begin{tabular}{|c|c|c|c|c|c|c|c|c|c|}
\hline & Industry & $\begin{array}{l}\text { Compensation } \\
\text { of employees }\end{array}$ & $\begin{array}{l}\text { Taxes on } \\
\text { production }\end{array}$ & $\begin{array}{l}\text { Subsidies on } \\
\text { production }\end{array}$ & $\begin{array}{l}\text { Net operating } \\
\text { surplus }\end{array}$ & $\begin{array}{l}\text { Net mixed } \\
\text { income }\end{array}$ & $\begin{array}{c}\text { Consumption } \\
\text { of fixed } \\
\text { capital }\end{array}$ & Value added & Gross output \\
\hline 1 & Agriculture & $\begin{array}{c}72,255,140 \\
(1.13)\end{array}$ & $\begin{array}{c}12,033,320 \\
(0.19)\end{array}$ & $\begin{array}{c}-8,330,602 \\
(-0.13)\end{array}$ & $\begin{array}{c}6,223,971,000 \\
(97.01)\end{array}$ & $\begin{array}{c}65,012,030 \\
(1.01)\end{array}$ & $\begin{array}{c}50,855,150 \\
(0.79)\end{array}$ & $\begin{array}{c}6,415,796,038 \\
(100)\end{array}$ & $19,204,942,655.41$ \\
\hline 2 & Fishing & $\begin{array}{c}10,557,110 \\
(2.34)\end{array}$ & $\begin{array}{c}440,335.3 \\
(0.09)\end{array}$ & $\begin{array}{c}-292,756.2 \\
(-0.06)\end{array}$ & $\begin{array}{l}436,125,700 \\
(96.49)\end{array}$ & $\begin{array}{c}2,703,238 \\
(0.59)\end{array}$ & $\begin{array}{c}2,446,571 \\
(0.54)\end{array}$ & $\begin{array}{c}4,519,801,98.1 \\
(100)\end{array}$ & $567,250,260.9$ \\
\hline 3. & $\begin{array}{c}\text { Food/ } \\
\text { beverage }\end{array}$ & $\begin{array}{c}226,730,400 \\
(2.69)\end{array}$ & $\begin{array}{c}33,005,090 \\
(0.39)\end{array}$ & $\begin{array}{c}-12,248,850 \\
(-0.15)\end{array}$ & $\begin{array}{c}8,048,809,000 \\
(95.41)\end{array}$ & $\begin{array}{c}94,373,290 \\
(1.12)\end{array}$ & $\begin{array}{c}45,572,020 \\
(0.54)\end{array}$ & $\begin{array}{c}8,436,240,950 \\
(100)\end{array}$ & $587,920,334.78$ \\
\hline \multirow[t]{2}{*}{4} & $\begin{array}{l}\text { Mining/ } \\
\text { quarrying }\end{array}$ & $\begin{array}{c}177,656,300 \\
(1.21)\end{array}$ & $\begin{array}{c}40,928,560 \\
(0.28)\end{array}$ & $\begin{array}{c}-708,251.1 \\
(-0.005)\end{array}$ & $\begin{array}{c}14,184,160,000 \\
(96.83)\end{array}$ & $\begin{array}{c}144,330,600 \\
(0.99)\end{array}$ & $\begin{array}{c}102,859,000 \\
\quad(0.70)\end{array}$ & $\begin{array}{c}14,649,226,208.9 \\
(100)\end{array}$ & $322,729,713,512.514$ \\
\hline & $\begin{array}{l}\text { Total of all } \\
\text { industries }\end{array}$ & $262,278,320$ & $86,407,305.3$ & $-21,580,459.3$ & $23,291,491,800$ & $306,419,158$ & $201,732,741$ & $24,126,748,865$ & $43,089,826,763.604$ \\
\hline
\end{tabular}

NB: Value added is calculated as the sum of compensation of employees, operating surplus, consumption of fixed capital, other taxes on products, and subsidies. 


\section{Multipliers}

Multipliers are derived based on direct and indirect effects arising from associate exogenous amendment in an industry's final demand. These multipliers which were estimated on the basis of the I-O analysis, are defined as the system of economic transactions that follow a disturbance in an economy. The Type I multipliers considers only the direct and indirect effects while the Type II multipliers consider both direct, indirect, and induced multipliers. The results of the multiplier coefficients are as presented in Table 7.

As presented in Table 7, different industry groups within the agricultural, fishing, food/beverage and mining/quarrying sectors have varying multiplier coefficients. This means their abilities to generate economic effects are different. The results explain that every $\$ 1$ additional demand for agriculture generates a total of \$ 1.76 and \$ 1.77 output and income respectively throughout the economy in 2010.

In other words, a 1dollar investment in the fishing industry will lead to a 2.89 and 3.19 increase in output and income (which is the highest when compared to other sectors) in the economy when both intermediate and final demand sectors (Type 11) are considered. Similarly, a 1dollar investment in the fishing industry will lead to a 2.11 and 2.22 increase in output and income in the economy when only the intermediate sectors (Type 1) are considered. Hence, the output and income in the fishing industry make up 27.93 and 29.24 percent of total domestic production. This implies that the fishing industry does not only represents a major socio-economic sector, but also is one of the major contributors to Nigeria's

Table 7. Multiplier coefficients.

\begin{tabular}{|c|c|c|c|c|c|c|c|c|}
\hline \multicolumn{9}{|c|}{ Total output multipliers } \\
\hline \multirow{2}{*}{ Sector } & \multicolumn{8}{|c|}{ Nigeria's input output } \\
\hline & INITIAL & FIRST & INDUS & TOTAL & CONS'M & TOTAL & TYPE I & TYPE II \\
\hline Agric & 1.000 & 0.383 & 0.376 & 1.758 & 0.653 & 2.411 & 1.758 & 2.411 \\
\hline Fishing & 1.000 & 0.613 & 0.505 & 2.118 & 0.772 & 2.890 & 2.118 & 2.890 \\
\hline Food/bev. & 1.000 & 0.399 & 0.326 & 1.726 & 0.910 & 2.635 & 1.726 & 2.635 \\
\hline Mining/Q & 1.000 & 0.440 & 0.363 & 1.803 & 0.609 & 2.412 & 1.803 & 2.412 \\
\hline \multicolumn{9}{|c|}{ Total income multipliers } \\
\hline \multirow{2}{*}{ Sector } & \multicolumn{8}{|c|}{ Nigeria's input output } \\
\hline & INITIAL & FIRST & INDUS & TOTAL & CONS'M & TOTAL & TYPE I & TYPE II \\
\hline Agric & 0.141 & 0.053 & 0.055 & 0.249 & 0.109 & 0.357 & 1.769 & 2.540 \\
\hline Fishing & 0.133 & 0.087 & 0.074 & 0.294 & 0.128 & 0.423 & 2.222 & 3.190 \\
\hline Food/bev. & 0.243 & 0.055 & 0.049 & 0.347 & 0.151 & 0.498 & 1.430 & 2.063 \\
\hline Mining/Q & 0.107 & 0.072 & 0.053 & 0.232 & 0.101 & 0.334 & 2.178 & 3.127 \\
\hline
\end{tabular}

Input-output analysis result from Eurostat database. 
GDP in terms of output and income to Nigeria's economy. The economic meaning of this is that salaries \& wages received by employees in the fishing industry have gone through more rounds of subsequent purchases than any other industry. In general, induced effects added by employee income are more than the total direct and indirect effects indicated by the Type I multiplier. Hence, the resultant effect from the protectionist trade measures in fish production (import quota) introduced since the first quarter of 2014 has stimulated the country's self-sufficiency through a 25 percent annual fish import cut.

Currently in Nigeria, fish production by artisanal fishers dominates fish production in Nigeria contributing about $85 \%$ of fish production, since aquaculture that could compliment the fisheries is not well developed. This sector employs over eight million fishermen, and regarding eighteen million individuals have interactions in fish process, distribution and selling that accounts for over eightieth of the entire annual domestic fish production [15]. Hence, the fishing industry represents the highest Type I and Type II multiplier coefficients when both output and income are considered.

\section{Conclusions and Suggestions for Further Studies}

An input-output multiplier approach was used to measure the economic impacts of mining/quarrying and agricultural related industries. The Type I and II multipliers were derived as measures of direct, indirect and induced effects emanating from a change in final demand. Mining/quarrying as a single sector had a Type I multiplier of 1.80 and 2.17 for both output and income respectively and a Type II multiplier of 2.41 and 3.12 for both output and income respectively. Similarly, the agricultural related sector (fishing) was identified to have the highest contributions (2.11 and 2.89 as well as 2.22 and 3.19) in both Types I and II multipliers for both output and income respectively. The different industries had varying multiplier coefficients, which means their abilities to generate economic activities also vary.

The findings of our research were limited by the availability of an up-to-datedata and therefore the present study has given more focus on the application of the methodology and opines on the need for further studies to adopt this study using the most recent data available, then make comparison in order to understand the changes in the multiplier effects occurring over time. Further research is also needed to address the product-wise economic impacts in addition to the aspects such as employment multipliers, import leakage and changing patterns of inter-industry dependence over time as the present study focused on industry-wise economic impacts, as well as the income and output multipliers.

\section{Availability of Data and Materials}

Data for the study were obtained online from Eurostat database. These datasets used and/or analyzed in the study are available from the corresponding author on reasonable request. 


\section{Acknowledgements}

Special acknowledgement goes to the Almighty God for his grace and DAAD for their financial support and encouragement.

\section{Funding}

The major funding source for this paper was obtained from Deutscher Akademischer Austauschdienst German Academic Exchange Service (DAAD) (Grant No. 91677725).

\section{Authors' Contributions}

The corresponding author, MOE handled the research methodology, analysis and interpretation, while the co-author, CUO, conceptualized the research work, literature and validated the results, and NJN proffered suggestions for further studies. All authors read and approved the final manuscript.

\section{Conflicts of Interest}

The authors declare no conflicts of interest regarding the publication of this paper.

\section{References}

[1] Federal Republic of Nigeria (2014) Intended Nationally Determined Contributions (INDC) Second National Communication Report. 1-23.

[2] National Bureau of Statistics (2018) Nigerian Gross Domestic Product Report Q4 2018. 1-141.

[3] Annual Abstract of statistics (2015) Annual Abstract of Statistics. http://www.nigerianstat.gov.ng

[4] Leontief, W. (1953) The Structure of the American Economy. Oxford University Press, New York.

[5] Frechtling, C. and Horvath, E. (1999) Estimating the Multiplier Effects of Tourism Expenditures on a Local Economy through a Regional Input-Output Model. Journal of Travel Research, 37, 324-332. https://doi.org/10.1177/004728759903700402

[6] United Nations (1993) System of National Accounts. Statistics Division Department for Economic and Social Affairs Statistics Division, New York. http://unstats.un.org/unsd/sna1993

[7] Eurostat (2008) Manual of Supply, Use and Input-Output Tables. Tech. Rep. No. L-2920. Luxembourg.

[8] Thijs, R. (2006) The Economics of Input-Output Analysis. Cambridge University Press, Cambridge.

[9] Krumme, G. (2009) http://faculty.washington.edu/krumme/systems/ioquations.html

[10] Diekmann, A. (2002) The Use of Energy in Transport-Past and Future Trends and Implications. 14th Input-Output Conference, Montreal, 10-15 October 2002, 1-10.

[11] Weisbrod, G. and Weisbrod, B. (1997) Assessing the Economic Impact of Transportation Projects How to Choose the Appropriate Technique for Your Project [Brochure]. Economic Development Research Group Transportation Research Board, 
Washington DC.

[12] Oosterhaven, J. and Stelder, D. (2000) On the Use of Gross versus Net Multipliers with a Bi-Regional Application on Dutch Transportation. 13th International Conference on Input-Output Techniques, Macerata, August 2000, 1-11.

[13] Hirschman, A.O. (1958) The Strategy of Economic Development. Yale University Press, New Haven.

[14] United Nations (1999) Handout of Input-Output Table Compilation and Analysis [Brochure]. Department for Economic and Social Affairs Statistics Division, New York, UN Publications No. E99.XVII.9.

[15] Federal Department of Fisheries (FDF) (2008) Fishery Statistics of Nigeria. 4th Edition, Federal Department of Fisheries, Abuja, 49. 